\title{
Hábitos de ingesta y actividad física en escolares, según tipo de establecimiento al que asisten
}

\author{
Raquel Burrows A, Erick Díaz Ba, Vito Sciaraffia M, \\ Vivien $\mathbf{G}$ attas $\mathbf{Z}^{\mathbf{b}}$, Ana Montoya $\mathbf{C}^{\mathrm{c}}$, Lydia Lera $\mathbf{M}^{\mathrm{d}}$. \\ Dietary intake and physical activity \\ in school age children
}

Background: The increased infantile obesity rates are related to faulty dietary intake (DI) and physical activity (PA) habits, that are probably related to a prolonged stay at school during the day. Aim: To investigate DI and PA among elementary and high school students and their association with type of school that they attend. Material and Methods: Quality of DI and PA was assessed, using specially designed questionnaires, in 1136 elementary school and 1854 high school children attending public schools managed by city halls (ME), subsidized private (SE) and private (PE) of the Metropolitan Region. The responses to the questionnaires, were qualified using a numeric scale that ranged from 0 to 10 points. A higher score indicated a better habit. Results: Percentile 25 ( $\mathrm{p} 25$ th) PA score was 4 and 3 in elementary and high school children respectively and the p 25 th for DI were 5.7 and 4.3, respectively. No differences in DI scores, according to the type of school, were observed. However, physical activity scores were significantly lower in children and adolescents from ME schools than from PE schools. Sixty percent of ME schools had less than 2 hours per week of programmed physical activity compared to more than 3 hours, in 70\% of PE schools. Elementary school children and high school adolescents expended 8 and 11 hours per day, respectively, in minimum expenditure activities. Conclusions: There is a greater deterioration of PA than DI among school age children and adolescents. Those attending ME schools have the worst physical activity scores. This fact must be addressed in future healthy lifestyle encouragement policies (Rev Méd Chile 2008; 136: 53-63).

(Key words: Child behavior; Child preschool; Exercise)
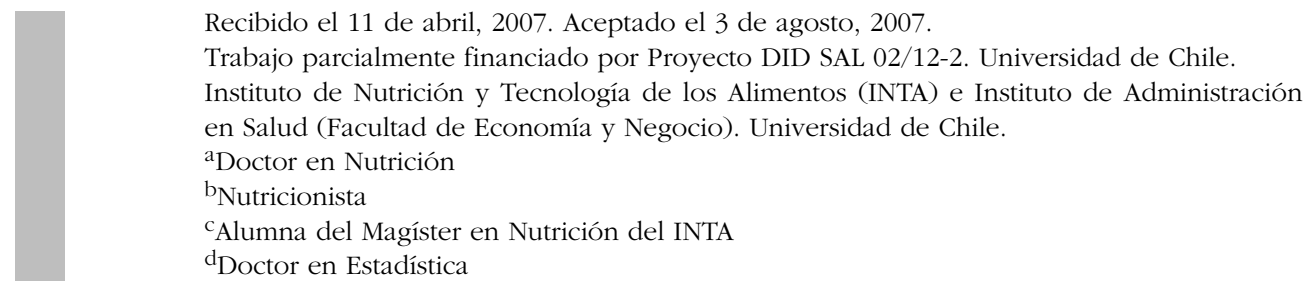

Correspondencia a: Dra. Raquel Burrows Argote. INTA. Uni- 
$\mathrm{L}^{\mathrm{a}}$ a dieta y los estilos de vida occidentales, estarían regulando la expresión genética de las enfermedades crónicas asociadas a la obesidad ${ }^{1-3}$ En Chile, el aumento de la obesidad y de sus comorbilidades (hipertensión arterial, DM2 y cardiopatías isquémicas), se relaciona con cambios en la ingesta y actividad física, propios del modelo de desarrollo económico ${ }^{4}$. En niños, la obesidad se ha cuadruplicado en los últimos 20 años ${ }^{5-8}$. En primero básico, aumentó de 5,8\% a 17\% entre los años 1987 y 2000 y en escolares aumentó de 4,6\% a 24,0\% (prepúberes) y de $2.3 \%$ a 17\% (púberes), entre los años 1986 y 1998 para posteriormente estabilizarse ${ }^{5,8}$. En preescolares en cambio, la obesidad aumentó de 5,7\% a $11,6 \%$ entre los años 2004 y $2006^{6,7}$. Por otro lado, estudios clínicos señalan que 30\% de los niños que consultan por obesidad, presentan el síndrome metabólico de resistencia insulínica (SMRI) 9 .

La ingesta aumentada de alimentos hipercalóricos y la jornada escolar completa (JCE), podrían estar contribuyendo al aumento del sobrepeso. La dieta del chileno se "occidentalizó" entre la década del 70 y la del 90 con mayor consumo de grasas $(13,9 \mathrm{k} /$ pers/ año en 1975 a 16,7 k en 1995) y alimentos elaborados (30,2 k/pers/año en 1975 a 39,2 k en 1995) y un bajo consumo de pescado, legumbres y verduras, con diferencias por nivel socioeconómico (NSE) en el consumo de alimentos altamente calóricos ${ }^{4,10}$. Estudios recientes en escolares de Santiago, muestran una ingesta diaria alta de alimentos calóricos (343 a $460 \mathrm{~g}$ ) y baja de frutas y verduras (197 a 270 g) y de lácteos (240 a $308 \mathrm{~g})^{11}$. Por otras parte, los escolares de NSE bajo consumen una mayor cantidad de alimentos calóricos y una menor cantidad de lácteos que los de NSE medio alto, sin diferencias en el consumo de frutas y pescado que no superan los $200 \mathrm{~g}$ y los $9 \mathrm{~g} /$ día, respectivamente ${ }^{12,13}$.

La inactividad física también es un factor determinante del sobrepeso. En adultos, el sedentarismo (menos de 30 min semanales de ejercicios) aumentó entre 1988 y 1999 (de 55,4\% en los varones y $77,4 \%$ en las mujeres a $85 \%$ en ambos sexos $)^{4}$. En preescolares, predomina la actividad física sedentaria con más de 60\% del tiempo diario en actividades de gasto mínimo (dormir, ver televisión (TV), etc. $)^{14}$. En niños y adolescentes con sobrepeso, la inactividad física sería el factor preponderante en la retención calórica diaria ${ }^{15}$. En escolares de sexo femenino, se observó una relación directa entre la edad y la frecuencia de actividad física en los días de colegio, sin diferencias por NSE; pero hay un mayor tiempo de ver TV en las escolares de nivel medio que las de nivel bajo ${ }^{13}$.

El programa "Vida Chile" ha implementado una serie de políticas y programas tendientes a mejorar la calidad de la alimentación y de la actividad física, varias de ellas insertas como un plan piloto, en el sistema escolar público ${ }^{16,17}$. Sin embargo, la Reforma Educacional Chilena extendió la jornada escolar diaria a $8 \mathrm{~h}$ para mejorar la calidad de los aprendizajes, sin modificar los 90 min semanales de ejercicio programado, por lo que los escolares podrían permanecer 7 h o más, en actividades de gasto mínimo ${ }^{18}$. Sería importante conocer cómo interactúan ambos componentes del balance energético e identificar posibles diferencias por NSE, con el objeto de optimizar las intervenciones dirigidas a promover una vida saludable. El objetivo de este estudio fue investigar la calidad de los hábitos de ingesta y actividad física en escolares y su asociación con el tipo de establecimiento al que asisten.

\section{MATERIAL Y MÉTODO}

Entre los años 2003 y 2004, se sortearon aleatoriamente 11 establecimientos municipalizados (EM), subvencionados (ES) y privados (EP) de la Región Metropolitana (RM), considerando la proporcionalidad por sexo y tipo de establecimiento (según Compendio de Información del MINEDUC), con el objeto de seleccionar escolares de primero básico y medio. El tamaño muestral (3.000) se obtuvo por la metodología para muestras aleatorias considerando el universo de escolares de primero básico (91.354) y medio (90.958) de la RM y un intervalo de confianza de 99\%. Dos mil novecientos noventa escolares (1.136 niños y 1746 adolescentes) ingresaron al estudio, después de firmar con sus padres un consentimiento según norma del Comité de Ética del INTA.

Antropometría. Se midió el peso y la talla con una balanza electrónica de precisión $\left(\mathrm{SECA}^{\circledR}\right)$ con cartabón incluido (metodología de Frankfurt) y el perímetro de cintura (PC) por sobre el reborde de la cresta ilíaca, con cinta métrica de fijación automática $\left(\mathrm{SECA}^{\circledR}\right)^{19}$. La adecuación ponderal se evaluó por el puntaje $\mathrm{z}$ del IMC del referente CDC-NCHS ${ }^{20}$. 
Hábitos. La calidad de los hábitos de ingesta y actividad física se evaluaron por dos encuestas ad-hoc. Ambas fueron respondidas por los adolescentes y, en el caso de los niños, ellos fueron ayudados por sus madres o guardadoras.

La encuesta de actividad física (HAF) (Anexo 1) evaluó: 1. Horas diarias acostado. 2. Horas diarias de actividades mínimas. 3. Número de cuadras caminadas diariamente. 4. Horas diarias de juegos recreativos, y 5 . Horas semanales de ejercicios o deportes programados.

La encuesta de ingesta (Anexo 2) evaluó: 1. número de comidas diarias. 2. Calidad de los alimentos o preparaciones del desayuno y once. 3 . Calidad de los alimentos del almuerzo y cena, 4. Calidad de los alimentos de la colación, y 5 . Calidad de los alimentos extras. En cada ítem se incluyeron todos los alimentos que consumen a lo menos una vez por semana y se ponderan de acuerdo a las veces que lo consumen en la semana. Los alimentos ricos en grasa y azúcar, se calificaron con puntaje 0 , los elaborados pero normo graso con puntaje 1 y los ricos en fibra y bajos en grasa saturadas o en azúcares con puntaje 2 .

Cada uno de los cinco aspectos de cada encuesta se ponderó con un puntaje de 0 a 2 , por lo que el puntaje total fluctuó entre 0 a 10 puntos, señalando en sentido creciente una mejor calidad del hábito.

Estadística. Los datos fueron procesados en una planilla EXCEL y en STATA 9.0 Se utilizó la distribución percentilar para estudiar asociaciones de la calidad de los hábitos ( $\leq \mathrm{p} 25,>\mathrm{p} 25$ y $<\mathrm{p} 75$ y $\geq p$ 75) con el tipo de establecimiento. Se usó Chi 2 para estudiar asociaciones y análisis de varianza de clasificación simple y prueba de comparación múltiple de Tuckey para comparar variables continuas. Se consideró significativo un $\mathrm{p}<0,05$.

\section{Resultados}

La Tabla 1 muestra las características generales de los escolares de básica por tipo de establecimiento

Tabla 1. Características generales de los escolares de básica, según tipo de establecimiento

\begin{tabular}{|c|c|c|c|c|}
\hline & Privados & $\begin{array}{l}\text { de Establecimiento } \\
\text { Subvencionados }\end{array}$ & Municipalizados & \\
\hline Mujeres (n) & $(146)^{1}$ & (90) & (243) & $\mathrm{P}^{2}$ \\
\hline Edad (años) & $7,0 \pm 0,^{*} \mathrm{~S}, \mathrm{M}$ & $6,6 \pm 0,5^{2}$ & $6,7 \pm 0,5$ & $<0,05$ \\
\hline IMC (ptje z) & $0.75 \pm 0,97^{*} \mathrm{~S},{ }^{* * \mathrm{M}}$ & $1,03 \pm 1,10$ & $1,05 \pm 1,14$ & $<0,05$ \\
\hline Cintura $(\mathrm{cm})$ & $58,1 \pm 5,7$ & $57,2 \pm 5,7$ & $57,5 \pm 5,7$ & n.s. \\
\hline HAF (puntaje) $^{3}$ & $4,8 \pm 1,4$ & $4,8 \pm 1,5$ & $4,1 \pm 1,3^{* * * *} \mathrm{P}, \mathrm{S}$ & $<0,0001$ \\
\hline HI (puntaje) $)^{4}$ & $6,4 \pm 1,0$ & $6,4 \pm 0,8$ & $6,5 \pm 1,1$ & n.s \\
\hline A G M $(\mathrm{h} / \text { día })^{5}$ & $8,4 \pm 1,5$ & $8,5 \pm 1,4$ & $8,6 \pm 1,2$ & n.s \\
\hline Varones (n) & (168) & (163) & (226) & \\
\hline Edad (años) & $7,0 \pm 0,7^{* *} \mathrm{~S}, \mathrm{M}$ & $6,6 \pm 0,3$ & $6,7 \pm 0,4$ & $<0,01$ \\
\hline IMC (puntaje z) & $0,91 \pm 1,08$ & $0,98 \pm 1,23$ & $1,26 \pm 1,47^{*} \mathrm{~S}, \mathrm{P}$ & $<0,05$ \\
\hline Cintura $(\mathrm{cm})$ & $58,4 \pm 5,1$ & $56,7 \pm 5,7$ & $57,7 \pm 6,1$ & n.s. \\
\hline HAF (puntaje) & $4,8 \pm 1,5$ & $5,1 \pm 1,6$ & $4,3 \pm 1,4^{* * *} S, P$ & $<0,0001$ \\
\hline HI (puntaje) & $6,3 \pm 1,1$ & $6,3 \pm 1,0$ & $6,3 \pm 0,9$ & n.s \\
\hline A G M (h / día $)$ & $8,6 \pm 1,4$ & $8,6 \pm 1,2$ & $8,7 \pm 1,4$ & n.s \\
\hline
\end{tabular}

${ }^{1}$ número de casos

${ }^{2}$ Significación según prueba multivarianza

${ }^{3}$ Hábitos de ingesta

${ }^{4}$ Hábitos de actividad física

${ }^{5}$ Horas de actividad de gasto mínimo

Significación según post Tuckey *p $<0,05 * * p<0,01 * *$ p $<0,001$. 
y sexo. Los escolares de EM muestran un mayor $\mathrm{z}$ IMC ( $p<0,05)$ y menores puntajes en los HAF $(<0,001)$ que los de ES y EP, sin diferencias en los puntajes de los $\mathrm{HI}$ ni en las horas diarias de actividades de gasto mínimo (clases, tareas, estudio, computador o TV). En los escolares de media (Tabla 2), las mujeres de EP, mostraron un menor z IMC (p <0,01) que las de ES y EM. En ambos sexos, hubo un menor PC ( $p<0,05$ y $p<0,001$ en varones y mujeres, respectivamente) y un mayor puntaje de los HAF ( $\mathrm{p}<0,001)$ en los escolares de $\mathrm{EP}$, que en los de EM y ES. No hubo diferencias por establecimiento en el puntaje de los HI, excepto en las mujeres de media, donde las de EM tienen un menor puntaje que los otros dos grupos (EP y ES). No hubo diferencias por tipo de establecimiento, en las horas diarias de actividades de gasto mínimo.

Hubo diferencias $(\mathrm{p}<0,001)$ en los puntajes obtenidos al comparar los HAF con los HI (Figura 1). Los escolares se concentran por debajo del puntaje 6,0 en los HAF y por sobre 6,0 puntos, en los HI. De esta forma, el p 25 de los HAF fue 4,0 en los niños y 3,0 en los adolescentes; en tanto que el p 25 de los HI fue 5,7 y 4,3 respectivamente.

No hubo diferencias por establecimiento en los HI, excepto en las mujeres de media (Figura 2 ), donde hubo un menor porcentaje de adolescentes de EM con hábitos $\geq \mathrm{p} 75$ que las de EP y ES. Hubo diferencias por establecimiento, en los HAF ( $p<0,001$ ), tanto en básica como en media (Figura 3). El 62,4\% y 64,6\% de las niñas y niños respectivamente de $\mathrm{EM}$, versus $45,2 \%$ y $37,4 \%$ de las niñas y niños, respectivamente de $\mathrm{EP}$, tuvieron puntajes $\leq$ p 25 (Figuras 3 A y B). En los adolescentes, $58,3 \%$ de las mujeres y $23,4 \%$ de los varones de EM, versus $22,3 \%$ y $14,5 \%$ de las mujeres y varones, respectivamente de EP, tuvieron puntajes $\leq \mathrm{p} 25$ (Figura $3 \mathrm{C}$ y D). Por otra parte, $36,8 \%$ de las adolescentes y 50,0\% de los adolescentes de EP tienen $\mathrm{HAF} \geq \mathrm{p} 75$ versus $10,5 \%$ y $36,1 \%$ de los $\mathrm{EM}$, respectivamente. Hubo diferencias ( $p<0,0001)$ por establecimiento, en las horas semanales de AF programada (Figura 4. En

Tabla 2. C aracterísticas generales (promedio y d.e.) de la muestra de escolares de media, según tipo de establecimiento

\begin{tabular}{|c|c|c|c|c|}
\hline & Privados & Subvencionados & Municipalizados & $\mathrm{p}^{2}$ \\
\hline Mujeres (n) & $(220)^{1}$ & (83) & $(456)$ & \\
\hline Edad (años) & $15,0 \pm 0,4^{* *} \mathrm{~S}, \mathrm{M}$ & $14,6 \pm 0,6$ & $14,7 \pm 0,6$ & $<0,01$ \\
\hline IMC (ptje z) & $0,38 \pm 0,75^{* * * *} \mathrm{~S},{ }^{* * *} \mathrm{M}$ & $0,89 \pm 1,11$ & $0,75 \pm 0,88$ & $<0,001$ \\
\hline P. Cintura $(\mathrm{cm})$ & $74,1 \pm 8,0 * * * \mathrm{~S}, \mathrm{M}$ & $80,1 \pm 10,9$ & $78,3 \pm 8,2$ & $<0,001$ \\
\hline HAF $(p t j e)^{3}$ & $4,9 \pm 1,7^{* * * *} \mathrm{~S}, \mathrm{M}$ & $3,5 \pm 1,8$ & $3,4 \pm 1,6$ & $<0,001$ \\
\hline HI $(\text { ptje })^{4}$ & $5,3 \pm 1,5$ & $5,5 \pm 1,3$ & $5,0 \pm 1,2 * \mathrm{P}, \mathrm{S}$ & $<0,05$ \\
\hline A G M $(\mathrm{h} / \text { día })^{5}$ & $11,5 \pm 2,0$ & $11,2 \pm 1,7$ & $11,2 \pm 1,9$ & n.s \\
\hline Varones (n) & (200) & (143) & $(752)$ & \\
\hline Edad (años) & $15,0 \pm 0,4$ & $14,8 \pm 0,34^{* * *} \mathrm{P}, \mathrm{M}$ & $15,0 \pm 0,40$ & $<0,01$ \\
\hline IMC (ptje z) & $0,45 \pm 0,81$ & $0,49 \pm 0,98$ & $0,58 \pm 1,07$ & n.s. \\
\hline P. Cintura $(\mathrm{cm})$ & $75,8 \pm 7,6^{*} \mathrm{M}$ & $76,1 \pm 9,2$ & $77,5 \pm 9,6$ & $<0,05$ \\
\hline HAF (ptje) & $5,4 \pm 1,7^{* * *} \mathrm{~S}, \mathrm{M}$ & $5,0 \pm 1,7$ & $4,8 \pm 1,7$ & $<0,001$ \\
\hline HI (ptje) & $5,2 \pm 1,3$ & $5,0 \pm 1,2$ & $5,0 \pm 1,2$ & n.s. \\
\hline A G M (h / día) & $11,7 \pm 2,1$ & $11,7 \pm 2,0$ & $11,6 \pm 2,1$ & n.s \\
\hline
\end{tabular}

${ }^{1}$ Número de casos

${ }^{2}$ Significación según prueba multivarianza

${ }^{3}$ Horas de Actividad de Gasto Mínimo

${ }^{4}$ Hábitos de Ingesta

${ }^{5}$ Hábitos de actividad física

Significación según post Tuckey * $\mathrm{p}<0.05^{* *} \mathrm{p}<0.01^{* * *} \mathrm{p}<0.001$ 


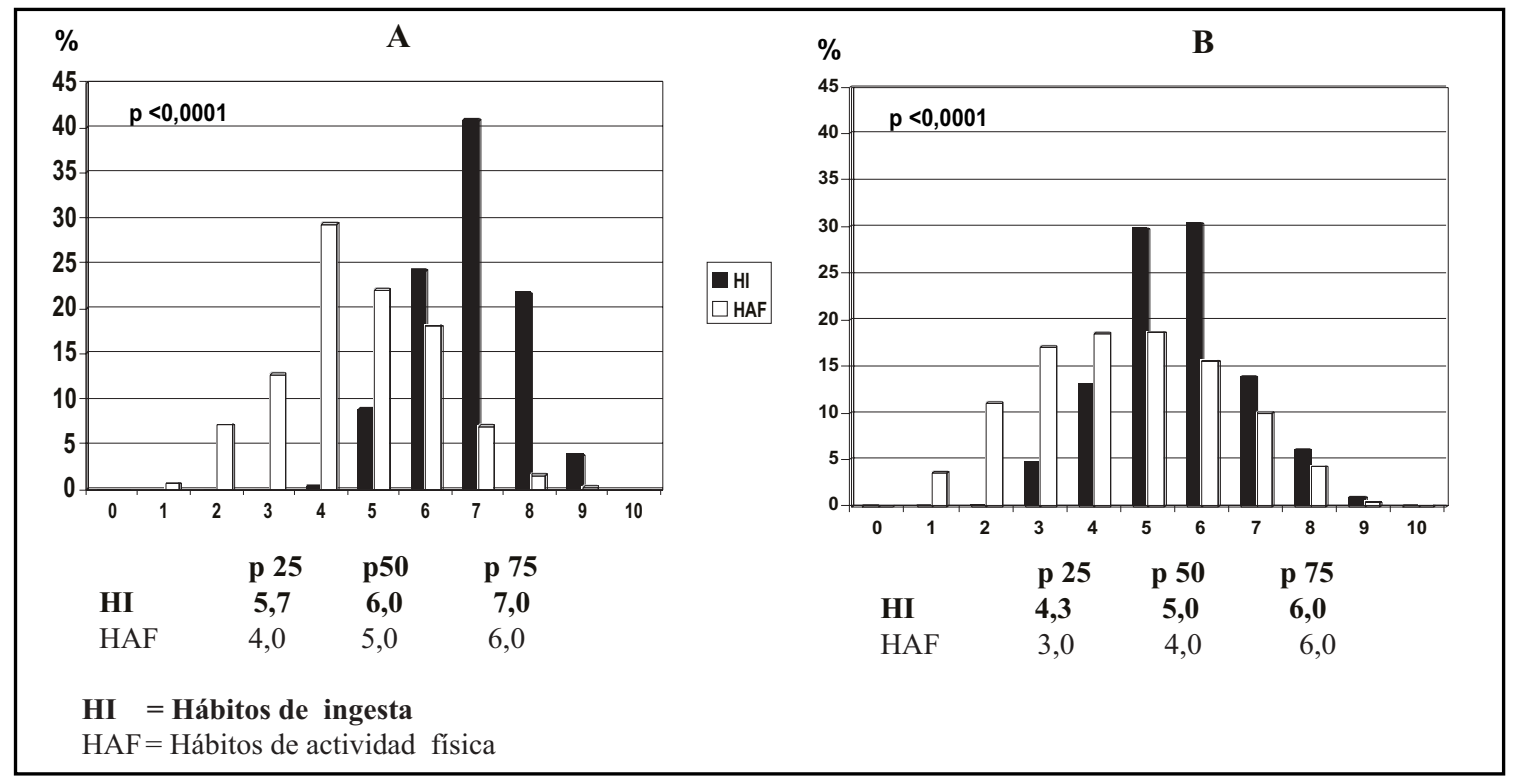

Figura 1. Distribución del puntaje de los hábitos de ingesta y actividad física en escolares de básica (A) y media (B).

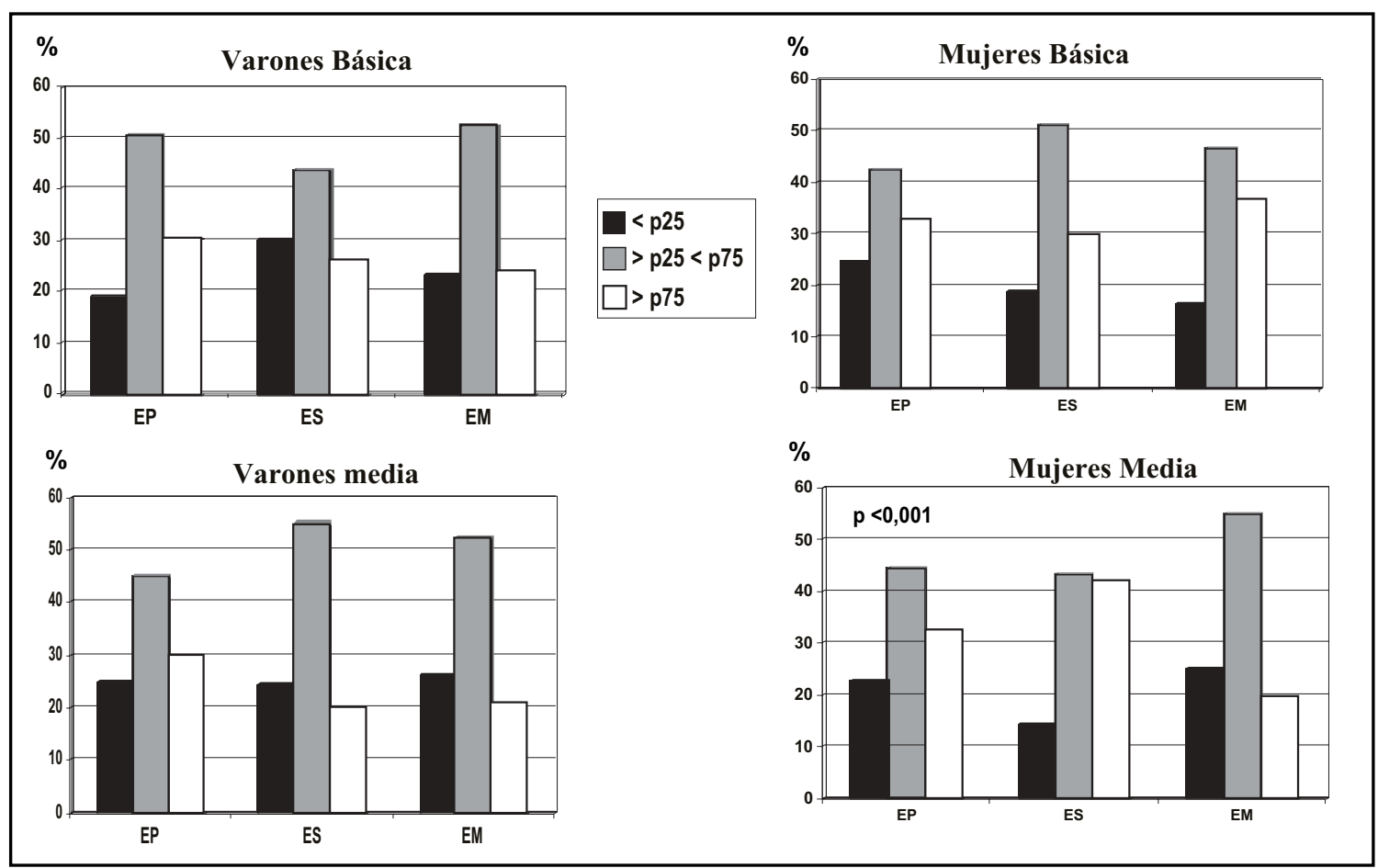

Figura 2. Distribución percentilar de los hábitos de ingesta según establecimiento. 


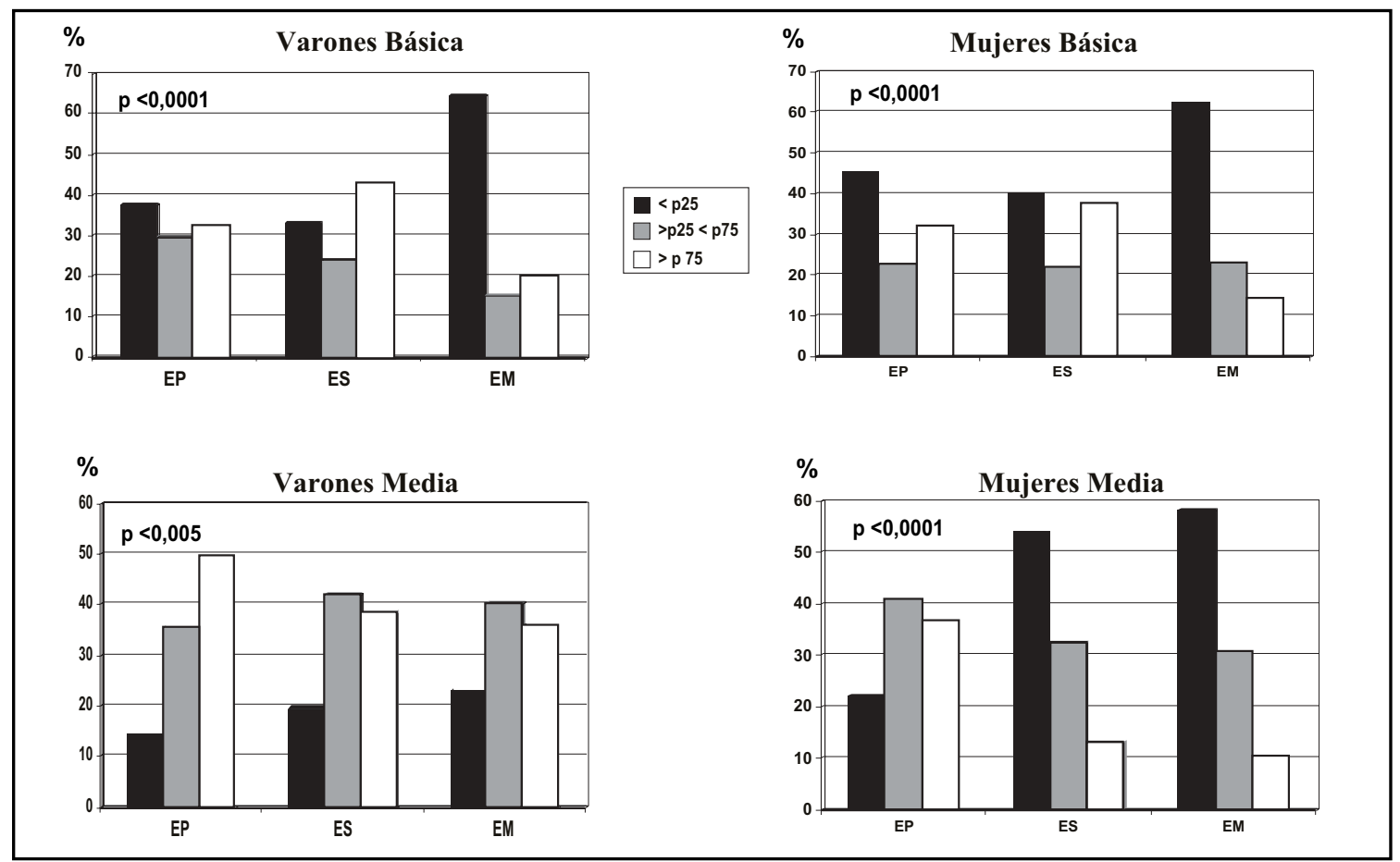

Figura 3. Distribución percentilar de los hábitos de actividad física según establecimiento.

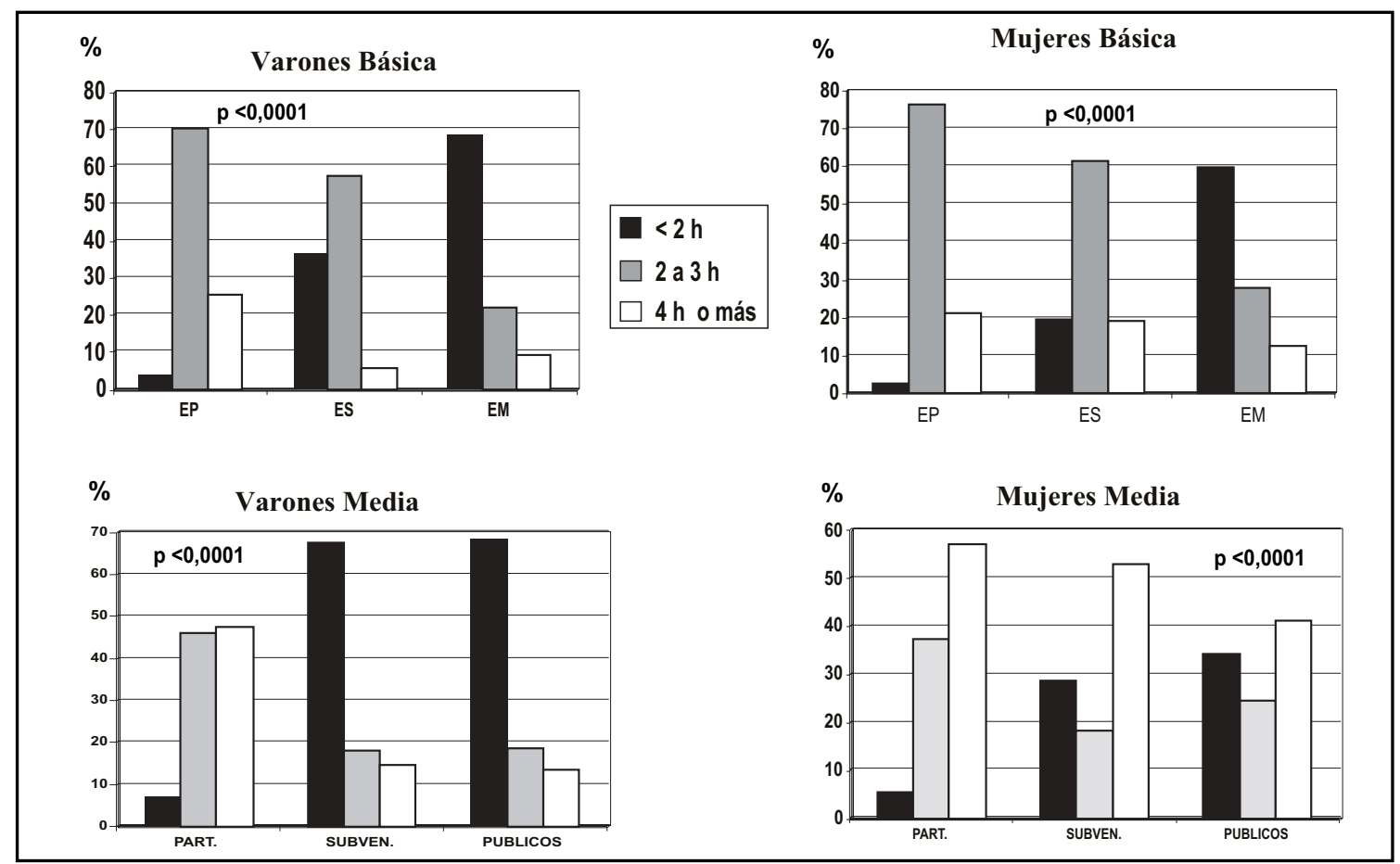

Figura 4. Actividad física programada (h semanas) según establecimiento. 

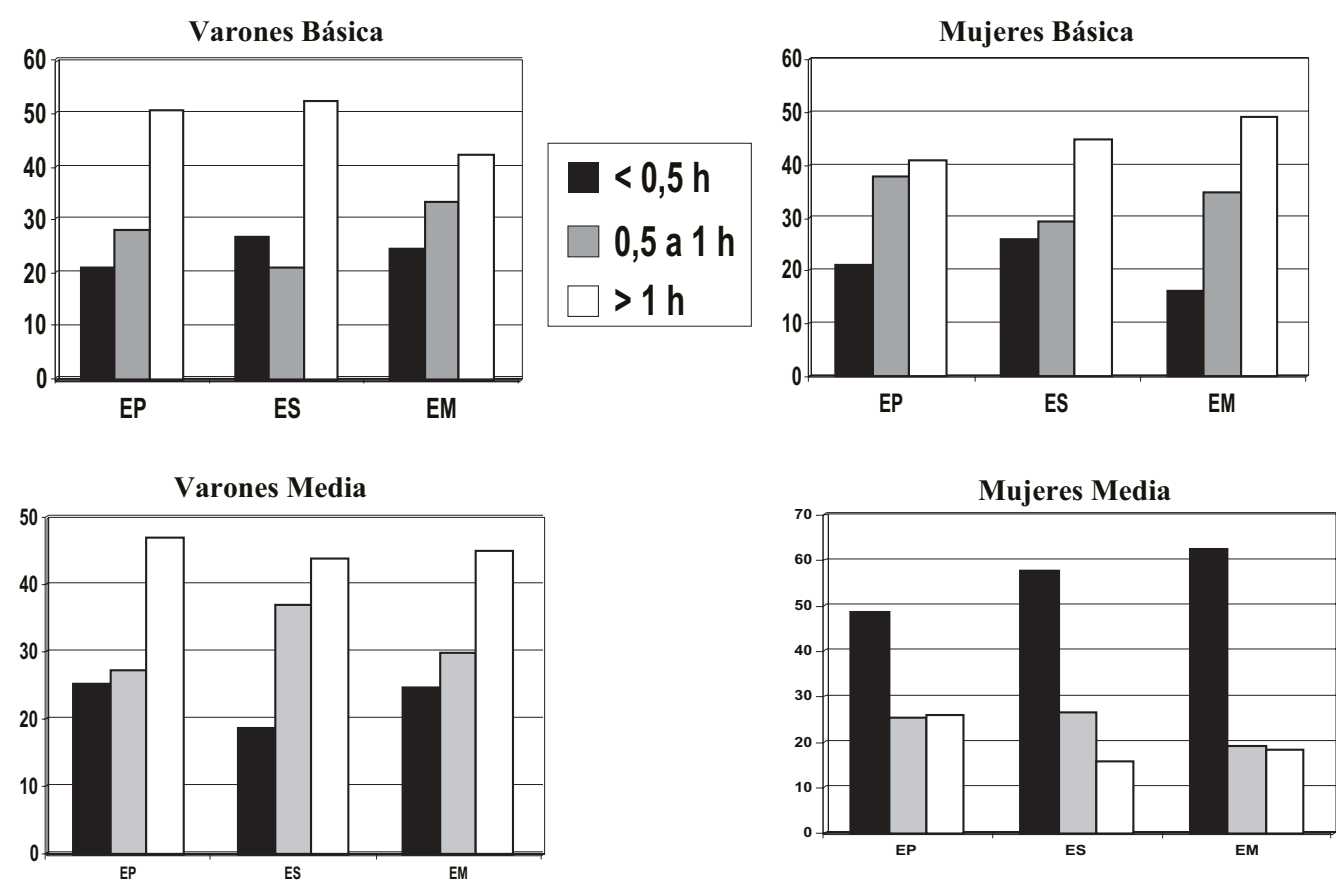

FiguRA 5. Actividad recreativa (h diarias), según establecimiento.

básica, 68,6\% de las niñas y 59,9\% de los niños de EM tenían menos de 2 h comparado con $4,1 \%$ y $2,5 \%$ de las niñas y niños, respectivamente, de $\mathrm{EP}$ (Figura 4 A y B). En media, 68,2\% de las mujeres y $34,3 \%$ de los varones de EM tenían menos de $2 \mathrm{~h}$ versus $5,5 \%$ y $6,8 \%$ de las mujeres y varones, respectivamente, de EP (Figura $4 \mathrm{C}$ y D). Por otra parte, $47,3 \%$ y $57,0 \%$ de las mujeres y varones, respectivamente, de $\mathrm{EP}$, tenían $4 \mathrm{~h}$ o más de ejercicio programado versus $13,4 \%$ y $41,2 \%$, respectivamente, en los EM.

No hubo asociación entre el tiempo diario de juego recreativo (Figura 5) y el tipo de establecimiento, excepto en las mujeres de media (Figura 5 C), donde hay un mayor porcentaje de mujeres que juegan menos de $0,5 \mathrm{~h}$ diarias entre las de EM y ES.

\section{DisCUSIÓN}

Se observan diferencias significativas al comparar la calidad de los hábitos de ingesta y actividad física en escolares de la RM. La mejor calidad de la ingesta, podría estar reflejando el impacto de las políticas y programas para promover una alimentación saludable, implementadas durante la última década ${ }^{17,21}$. Las guías alimentarias, los programas de alimentación de la Junta Nacional de Auxilio Escolar y Becas (JUNAEB) y Junta Nacional de Jardines Infantiles (JUNJI), el trabajo de grupos académicos con el Ministerio de Salud y Ministerio de Educación para elaborar programas y campañas para mejorar la calidad de la alimentación y con la industria alimentaria para mejorar la oferta de alimentos saludables, son parte de las acciones implementadas. Sus retornos parecieran estar favoreciendo en forma similar a la población escolar, ya que no se observan diferencias en la calidad de la ingesta por tipo de establecimiento.

No ocurre lo mismo con los hábitos de actividad física, que muestran diferencias significativas entre establecimientos municipalizados y privados. Mientras $70 \%$ de los niños y $90 \%$ de los adolescentes de establecimientos privados tienen $3 \mathrm{~h}$ semanales $\mathrm{o}$ más de ejercicio programado, 60\% de los establecimientos municipalizados, tienen sólo los 90 min del programa escolar. Se ha demostrado que sujetos que practican menos de $2,5 \mathrm{~h}$ semanales de actividad física moderada o vigorosa, tienen un aumento en la 
prevalencia de DM2 (85\%), enfermedad coronaria diabética (92\%), infarto al miocardio (117\%) y mortalidad (41\%), en comparación con sujetos que mantienen $>2,5 \mathrm{~h}^{22-24}$. En niños también se describe un menor número de factores de riesgo cardiovascular (hipercolesterolemia, hipertensión arterial y grasa corporal aumentada), una mayor capacidad aeróbica y fuerza muscular y menor grasa abdominal, en aquellos que realizan 3 o más h semanales de ejercicio programado, comparados con aquellos que sólo practican los 90 min del programa escolar ${ }^{25,26}$. En población infantil, tanto la capacidad aeróbica como la fuerza muscular se han relacionado indirecta y significativamente con el riesgo cardiovascu$\operatorname{lar}^{27,28}$. El entrenamiento muscular promueve una mayor captación y transporte de glucosa y oxidación de lípidos, utilizándose terapéuticamente en la prevención y en el tratamiento de la IR, ${ }^{29}$. En niños obesos, el ejercicio de entrenamiento mejora la dilatación de la arteria braquial, aun cuando no se modifica el peso, el IMC ni la grasa corporal ${ }^{30}$

Si bien no hubo diferencias por establecimiento en las horas diarias dedicadas a actividades de gasto mínimo, estas superaron las $8 \mathrm{~h}$ en los niños $\mathrm{y}$ las $11 \mathrm{~h}$ en los adolescentes. Se ha demostrado una fuerte asociación entre la inactividad física y enfermedades como DM2 y cardiopatía isquémi$\mathrm{ca}^{22-24,29}$. El "fenotipo deficiente en ejercicio" se asocia fuertemente a la insulino resistencia (IR) y al daño cardiovascular y endotelial asociado. ${ }^{29}$ Estudios nacionales muestran una alta prevalencia de IR (52\%) asociada a un perfil de riesgo cardiovascular y de síndrome metabólico (30\%) en niños y adolescentes obesos 31,9 .

Es necesario considerar algunas limitaciones en este trabajo. Fue realizado en escolares de RM y no podría generalizarse a toda la población, sin embargo, la JEC y los 90 minutos semanales de ejercicio programado, responden a una política pública escolar nacional. Otra limitación, es que se ha evaluado la calidad más que la cantidad, tanto de la ingesta como de la actividad física. Sin embargo, los

\section{REFERENCIAS}

1. World Health Organization. Obesity: Preventing and managing the global epidemic. Report of a WHO consultation. Geneva: WHO, 1998. diferentes aspectos considerados por ambas encuestas, tienen relación con el riesgo cardiovascular y metabólico asociado a la frecuencia del consumo de alimentos elaborados ricos en grasa y azúcares, a la exposición prolongada a actividades de gasto mínimo y a la falta de ejercicio físico moderado o vigoroso $^{25,27,28,32}$. El propósito de este estudio no ha sido pronunciarse sobre aspectos cuantitativos de la ingesta, sino más bien cualitativos, relacionados con la frecuencia de consumo de los diferentes alimentos. Por otra parte, la encuesta de actividad física, ha considerado el tiempo destinado al ejercicio físico y a las actividades de gasto mínimo. Esta última, ha sido validada en niños de 6 a 10 años a través de sensores de movimiento, con una adecuada reproducibilidad $(0,69$ a 0,93$)$ y concordancia $(0,58$ a $0,83)^{33}$.

Los resultados encontrados sugieren que las políticas y programas dirigidos a mejorar la calidad de la alimentación estarían llegando a la población escolar, independientemente del tipo de establecimiento al que asistan, aun cuando éstas deben continuar y desarrollarse nuevos programas para seguir mejorándolos. La "Estrategia global contra la obesidad" (EGO Chile) incluye recomendaciones concretas para aumentar la actividad física en el ámbito de la salud, pero no hay políticas ni programas que resguarden en los escolares un ejercicio físico programado en calidad y cantidad, para una vida saludable. El sistema escolar contempla 90 min de ejercicio físico semanal, tiempo muy por debajo de la recomendación mínima de 3,5 h de actividad moderada o vigorosa para proteger del sistema cardiovascular ${ }^{22-24}$. El tiempo prolongado de las actividades de gasto mínimo y las diferencias por establecimiento en las horas semanales de ejercicio programado, señalarían la necesidad de implementar acciones que promuevan una actividad física saludable en cantidad y calidad al interior del sistema escolar. Esto, resguardaría una adecuada equidad en la prevención de las enfermedades crónicas asociadas a la obesidad y al sedentarismo.

2. Libman I, Arslanian SA. Type II Diabetes Mellitus: No longer just adults. Pediatrics Annals 1999; 28: 589-93.

3. Desmond W, Knowler WC, Smith C, Hanson RL et AL. The effect of indian or anglo dietary preferen- 
ce on the incidence of diabetes in Pima Indians. Diabetes Care 2001; 24: 811-6.

4. Vio F, Albala C. Obesidad en Chile: una mirada epidemiológica. En Albala C, Kain J, Burrows R, Díaz E. editores. Obesidad un desafío pendiente. Santiago: Editorial Universitaria, 2000: 31-43.

5. Kain J, Burrows R, Uauy R. Obesity trends in children and adolescents: Basic Determinants. En: Obesity in Childhood and adolescent. Ch. Chen and W. Dietz Ed. Lippincott Williams \& Wilkins (Nestle Nutrition Worshop Series Pediatric Program 49). 2002; 45-63.

6. Rojas J. UauY R. Evolución de las normas de alimentación y nutrición del Programa Alimentario y cambios en el estado nutricional de preescolares beneficiario de la JUNJI. Rev Chil Nutr 2006; 33: 91-101.

7. Kain J, Lera L, Rojas J, Uauy R. Obesidad en preescolares de la región metropolitana de Chile. Rev Méd Chile 2007; 135: 63-70.

8. Muzzo S, Cordero J, Ramírez I, Burrows R. Trend in nutritional status and stature among school age children in Chile. Nutrition 2004; 20: 867-973.

9. Burrows R, Weisstaub G, Ceballos Z, Gattás V, Leiva L, Lera L, Albala C. Síndrome metabólico en niños y adolescentes: asociación con sensibilidad insulínica, grado y distribución del sobrepeso. Rev Med Chil 2007; 135: 174-81.

10. Berríos X. Tendencia temporal de los factores de riesgo de enfermedades crónicas: la antesala silenciosa de una epidemia que viene. Rev Méd Chile 1997; 125: 1405-7.

11. Olivares S, Kain J, Lera L, Pizarro F, Vio F, Morón C. Nutritional status, food consumption and physical activity among chilean school children. A descriptive study. Eu J Clin Nutr 2004; 58: 1278-85.

12. Olivares S, Zacarías I, Lera L. Estado nutricional y consumo de alimentos seleccionados en escolares de la región metropolitana: línea base para un proyecto de promoción del consumo de pescado. Rev Chil Nutr 2005; 32: 102-8.

13. Olivares S, Bustos N, Lera L, Zelada ME. Estado nutricional, consumo de alimentos y actividad física en escolares mujeres de diferentes NSE de Santiago. Rev Méd Chile 2007; 135: 71-8.

14. Vásquez F, Salazar G. Patrón de actividad física en un grupo de preescolares obesos asistentes a jardines infantiles de la JUNJI, evaluados con sensor de movimiento. Rev Chil Nutr 2005; 32: 110-7.

15. Burrows R, Burgueño M, Gattas V, Barrera G, Leiva L. Características biológicas, familiares y metabó- licas de la obesidad infanto juvenil. Rev Méd Chile 2001; 129: 1155-62.

16. Proyecto Nacional para la Promoción de la salud "Vida Chile". Ministerio de Salud. Edición 2000.

17. www.minsal.cl/ici/nutricion/nutricion.html

18. www.mineduc.cl/index0/phpid_portal=21

19. Fernández JR, Reeden DT, Petrobielli A, Allison DB. Waist circunference percentiles in nationally representative sample of african-american, european-american and mexican american children and adolescent. J Pediatr 2004; 145: 439-44.

20. NCHS-CDC, diciembre 2002; http//.cdc.gov/ growthcharts.

21. Pizarro T. Intervención nutricional a través del ciclo vital para la prevención de las enfermedades crónicas no transmisibles. Rev Chil Pediatr 2007; 78: 76-83.

22. Hu FB,Manson Je, Stampfeer MJ, Colditz G, Liu S, Solomon CG, Willet WC. Diet, life style, and the risk of type 2 diabetes mellitus in women. N Eng J Med 2001; 345: 790-7.

23. Hu FB, Sigal RJ, Rich Edwards JW, Colditz GA, Liu S, SOlomon CG, Willet WC. Walking compared with vigorous physical activity and risk of type 2 diabetes in women. JAMA 1999; 282: 1433-9.

24. Hu FB, Stampfeer MJ, Colditz GA, Liu S, Solomon CG, Ascherio A et Al. Physical activity and risk of stroke in women. JAMA 2000; 283: 2961-7.

25. Ribeiro JC, Guerra S, Oliveira J, Texeira-Pinto A, Twist JWR, Duarte JA, Mota J. Physical activity and biological risk factors clustering in paediatric population. Prev Med 2004; 39: 596-601.

26. Ara i, Vicente-Rodríguez E, Jiménez RJ, Dorado C, Serrano SJA, Calbert JAL. Regular participation in sports is associated with enhanced physical fitness and lower fat mass in prepuberal boys. Int J Obe 2004; 28: 1585-93.

27. Wisloff U, Naijar SM, Ellingsen O, Harm PM, Swoap S, Al-Share Q et al. Cardiovascular risk factors emerge after artificial selection for low aerobic capacity. Science 2005; 307: 418-20.

28. Jurca R, Lamonte Mi, Barlow CE, Kampert JB, Church TS, Blair SN. Association of muscular streng with incidence of metabolic syndrome in men. Med Sci Sport Ex 2005; 1849-1855.

29. Hawley JA. Exercise as therapeutic intervention for the prevention and treatment of insulin resistance. Diab Met Res Rev 2004; 20: 383-93.

30. Watts K,Beye P, Siafarikas A, O'Driscoll G, Jones TW, Davis EA, Green DJ. Effects of exercise 
training on vascular function in obese children. J Pediatr 2004; 144: 620-5.

31. Burrows R, Burgueño M, Gattas V, Leiva L, Ceballos X, Guillier I et al. Perfil de riesgo cardiovascular en niños obesos con menor sensibilidad insulínica. Rev Méd Chil 2005; 133: 795-804.
32. Riccardi G, Giacco R, Rivellese AA. Dietary fat, insulin sensitivity and metabolic syndrome. Clin Nutr 2004; 23: 447-56.

33. Burrows R, Godard C, Rodríguez MP, Diaz N, Lera L, SAlazAR G. Validación de un test de actividad física en niños obesos y no obesos. Proyecto Nestlé Fundation. Rev Chil Nutr 2006; 33(2): 396 (No 38-O).

\section{Anexo 1.- Encuesta de H ábitos de Actividad Física}

\section{Tipo de Actividad: Horario}

1.- Acostado

2.- Actividades de gasto mínimo: sentado

en las siguientes actividades:
a) Clases
b) TV
c) Tareas o estudio
d) Computador o nintendo

3.- Caminar: Cuadras caminadas para trasladarse al colegio o a cualquier otro

lugar al que van rutinariamente)

4.- Juegos recreativos después de la jornada escolar (se excluyen los recreos)

Bicicleta, patines, fútbol, otros

5.- Actividades sistemáticas

a) Educación física

b) Gimnasia aeróbica

c) Fútbol

d) Tenis

e) Básquetbol

f) Otros

\section{Horas de actividad}

Total h diarias

Total h diarias
a)
b)
c)
d)

Total cuadras diarias

Total h diarias

Total h semanales
a)
b)
c)
d)
e)
f)
$0=<2$ horas
$1=2$ a 4 horas
$2=>4$ horas

\section{Puntaje \\ $0=\geq 12$ horas \\ $1=11-9$ horas \\ $2=\leq 8$ horas}

$0=\geq 10$ horas

$1=9-7$ horas

$2=\leq 6$ horas

$0=<5$ cuadras

$1=5$ - 15 cuadras

$2=>15$ cuadras

$0=<30$ minutos

$1=30-60$ minutos

$2=\geq 60$ minutos

Puntaje total: 0 a 10 Pts.

1. Se registra el horario habitual en que se acuesta y se levanta de lunes a viernes. Si duerme siesta diariamente tb. se registra.

2. Se registran las horas sentadas en actividades que son parte de la rutina diaria sistemática del niño.

3. Se registran las cuadras caminadas diariamente en actividades que son parte de la rutina diaria o que se repiten a lo menos 4 veces por semana

4. Se registra el tiempo diario dedicado a actividades recreativas al aire libre, después del horario escolar. Se pueden sumar las horas semanales y dividir por siete, en aquellos casos que el juego se produce algunos días de la semana.

5. Se registran las hrs. semanales de educación física más todas aquellas dedicadas a deportes o talleres deportivos, con horario y que se repite semanalmente. 


\section{Anexo 2.- Encuesta de $\mathrm{H}$ ábitos de Ingesta}

\begin{tabular}{|c|c|c|}
\hline 1.- ¿Cuántas comidas hace al día? & 0 & 2 comidas \\
\hline \multirow[t]{2}{*}{ Desayuno- Almuerzo- Once- Cena-Colación } & 1 & 3 comidas \\
\hline & 2 & $4-5$ comidas \\
\hline \multirow[t]{3}{*}{$\begin{array}{l}\text { 2.- Combinaciones } \\
\text { desayuno/onces }\end{array}$} & 0 & $\begin{array}{l}\text { Leche entera, pan amasado, hallulla, pan con } \\
\text { queso, mantequilla o mortadela, té o café puro, hot } \\
\text { dog, pizza, productos de pastelería, huevo frito, } \\
\text { manjar, paté, arrollado }\end{array}$ \\
\hline & 1 & $\begin{array}{l}\text { Leche semi descremada, marraqueta, galletas agua/ } \\
\text { soda, mermelada, palta, margarina, huevo duro, } \\
\text { jamón de pavo, queso blanco, cereal azucarado. }\end{array}$ \\
\hline & 2 & $\begin{array}{l}\text { Leche o yogurt descremada, fruta, Jugo de } \\
\text { fruta natural, pan integral, cereales naturales } \\
\text { o integrales, quesillo, tomate }\end{array}$ \\
\hline \multirow[t]{3}{*}{$\begin{array}{l}\text { 3.-Combinaciones } \\
\text { almuerzo/comida }\end{array}$} & 0 & $\begin{array}{l}\text { Pastas (ravioles, lasaña, otros), Tortillas, } \\
\text { Frituras, postres elaborados(flanes,gozzo } \\
\text { etc.), fruta en conserva, fruta con crema o } \\
\text { miel, hot dog, hamburguesa, pizza, pantrucas, } \\
\text { bebida gaseosa, huevo frito o revuelto }\end{array}$ \\
\hline & 1 & $\begin{array}{l}\text { Carnes, arroz, fideos, puré, pan, papas, } \\
\text { sopas, compotas de frutas, jaleas, yogurt, } \\
\text { jugo en polvo, bebida diet }\end{array}$ \\
\hline & 2 & $\begin{array}{l}\text { Ensaladas con verduras de todo tipo, } \\
\text { guisos de verdura c/ carnes, pescado, ave, } \\
\text { cazuela, carbonada, leguminosas, postres } \\
\text { de frutas, jugos naturales,agua }\end{array}$ \\
\hline \multirow[t]{3}{*}{ 4.- Colaciones } & 0 & $\begin{array}{l}\text { Papas fritas u otras, pasteles, chocolates o } \\
\text { confites, snack, . helados, alfajores, negritas, } \\
\text { bebidas gaseosas, sopaipillas }\end{array}$ \\
\hline & 1 & $\begin{array}{l}\text { Sándwich palta o pollo, galletas, nueces, } \\
\text { galletones, cereales, jugos azucarados, } \\
\text { bebida Light }\end{array}$ \\
\hline & 2 & Fruta, yogurt, huevo duro \\
\hline \multirow[t]{3}{*}{ 5.- Alimentos extras } & 0 & $\begin{array}{l}\text { Dulces, chocolates, papas fritas y similares, } \\
\text { bebidas gaseosas, productos de pastelería, } \\
\text { snack, helados, sopaipillas, picarones, } \\
\text { pasteles, sándwich jamón o queso }\end{array}$ \\
\hline & 1 & $\begin{array}{l}\text { Galletas, sándwich de ave, atún, mermelada, } \\
\text { pan solo }\end{array}$ \\
\hline & 2 & Fruta, jugos fruta, yogurt,agua \\
\hline
\end{tabular}

Ud debe marcar (subrayar) todos los alimentos que ha consumido a lo menos una vez a la semana, durante los últimos 3 meses.

Los alimentos que se consumen todos los días se marcan 3 veces

Los alimentos que se consumen 1 o 2 veces por semana se marcan sólo una vez

Los alimentos que se consumen 3 a 5 veces a la semana se marcan 2 veces

Nota: El puntaje de los ítems 2 a 5 , se calcula resolviendo en cada uno la fracción correspondiente. Se coloca el número de marcas (subrayados) en el denominador y los puntos acumulas por el total de marcas, en el numerador. De esa forma se obtiene un valor de la fracción que va de 0,0 a 2,0. 